\title{
A group intervention which assists patients with dual diagnosis reduce their drug use: a randomized controlled trial
}

\author{
W. JAMES, N. J. PRESTON, G. KOH, C. SPENCER, S. R. KISELY And D. J. CASTLE* \\ Alma Street Centre, Fremantle Hospital and Health Service, Fremantle, WA 6160, Australia; Primary Care \\ Mental Health Unit of the University of Western Australia; and Mental Health Research Institute and \\ University of Melbourne, Australia
}

\begin{abstract}
Background. There is a well-recognized association between substance use and psychotic disorders, sometimes described as 'dual diagnosis'. The use of substances by people with psychosis has a negative impact in terms of symptoms, longitudinal course of illness and psychosocial adjustment. There are few validated treatments for such individuals, and those that do exist are usually impracticable in routine clinical settings. The present study employs a randomized controlled experimental design to examine the effectiveness of a manualized group-based intervention in helping patients with dual diagnosis reduce their substance use.
\end{abstract}

Method. The active intervention consisted of weekly 90 -min sessions over 6 weeks. The manualized intervention was tailored to participants' stage of change and motivations for drug use. The control condition was a single educational session.

Results. Sixty-three subjects participated, of whom $58(92 \%)$ completed a 3-month follow-up assessment of psychopathology, medication and substance use. Significant reductions in favour of the treatment condition were observed for psychopathology, chlorpromazine equivalent dose of antipsychotics, alcohol and illicit substance use, severity of dependence and hospitalization.

Conclusions. It is possible to reduce substance use in individuals with psychotic disorders, using a targeted group-based approach. This has important implications for clinicians who wish to improve the long-term outcome of their patients.

\section{INTRODUCTION}

There is a well-recognized association between substance use and psychotic disorders, sometimes described as 'dual diagnosis'. Approximately one half of individuals in the community with a psychotic disorder have a substance use disorder, and the same proportion of those who abuse substances have a psychiatric disorder (Schneier \& Siris, 1987; Regier et al. 1990; Fowler et al. 1998; Siegfried, 1998). The special

\footnotetext{
* Address for correspondence: Professor David J. Castle, Mental Health Research Institute and University of Melbourne, 155 Oak Street, Parkville, Victoria 3052, Australia.

(Email: dcastle@mhri.edu.au)
}

needs of this group of patients have been increasingly recognized in Great Britain, the United States and Australia (Dawe \& Mattick, 1997; Department of Health, 2002). These include increased symptoms of depression, suicidal ideation and psychosis (Strakowski et al. 1994; Krausz et al. 1996; Dixon, 1999), reflected in higher admission rates to psychiatric units (Martinez-Aravelo et al. 1994).

There have been relatively few studies of the effectiveness of interventions specifically for substance use in people with psychotic disorders, but there is a general consensus that such interventions require an integrated approach addressing both issues (Drake et al. 1998; Drake 
\& Mueser, 2000; Department of Health, 2002). We are not aware of any published group-based randomized controlled trials specifically targeting substance use in people with psychosis. The virtues of a group-based approach include the positive impact of group belonging, the sharing of information about harm-reduction strategies by members of the group, and cost effectiveness.

Another line of research that impacted on the design of the current study, was that examining psychotic individuals' reasons for substance use. We believed that knowledge of individuals' reasons for use could be usefully employed both in engagement, and therapeutically in the groupbased intervention. A number of studies have investigated motivations for substance use in psychosis (Test et al. 1989; Dixon et al. 1991; Warner et al. 1994; Baigent et al. 1995; Mueser et al. 1995; Addington \& Duchak, 1997; Fowler et al. 1998; Spencer et al. 2002). These findings have not to our knowledge been explicitly applied to the treatment of substance use in patients with dual diagnosis. Spencer et al. (2002) found that 'dealing with negative affect' and 'enhancement' motives actually maintained substance use disorders and acted as the mediator between symptoms and psychological dependence, making them a legitimate target for therapeutic intervention.

The current study, therefore, aimed to evaluate the effectiveness compared to standard treatment, of a manualized group intervention explicitly addressing motivations for use, in helping patients with dual diagnosis reduce their substance use.

\section{METHOD}

\section{Subjects}

Participants were recruited from three major community mental health services in Western Australia (Fremantle Mental Health Service, Rockingham Kwinana Health Service and Peel Health Service). Inclusion criteria were: (1) an Axis I diagnosis (ICD-10; WHO, 1994) of a non-organic psychotic disorder; (2) currently using alcohol or illicit drugs; (3) a willingness to discuss drug use in a group setting; (4) ability to converse in English without an interpreter; (5) absence of developmental disability sufficient to impair participation in the group process and (6) meeting ICD-10 criteria for harmful use and/or dependence, and being rated as having on-going drug use on the Severity of Dependence Scale (SDS), the Drug Abuse Screening Test (DAST; Skinner, 1982), and/or Opiate Treatment Index (Darke et al. 1992). Subjects were excluded from the study if they were already receiving treatment for substance use from another agency, or had previously received any group-based treatment for their substance misuse or psychosis. Participants were paid AUS\$20 for each follow-up assessment they completed. The research ethics committee of Fremantle Hospital approved the protocol, including the payment procedure. Each participant signed a consent form after being informed about the purpose of the study.

Subjects were drawn from a service that operates within a case management model, which assisted in ensuring high attendance rates amongst participants. Case managers were encouraged to follow-up the progress of their clients during the course of the treatment and to encourage attendance at the groups. All usual clinical interventions were offered irrespective of allocation to case or control group.

\section{Measures}

The following domains were assessed at baseline and at three month follow-up after conclusion of the group: (1) psychopathology; (2) quantity of drug use; (3) level of drug and alcohol abuse and dependence. All instruments were recommended by an Australian review of assessment instruments for alcohol and drug problems and psychiatric disorders (Dawe et al. 2002).

Psychopathology was measured using the Brief Psychiatric Rating Scale (BPRS; Overall \& Gorham, 1962), and the self-reported Brief Symptom Inventory (BSI; Derogatis, 1993). The total score of the BPRS and the Global Severity Index (GSI) from the BSI were used as global measures of psychopathology. The independent rater (N.P.) was trained on the BPRS and had scored within acceptable limits from two 'gold standard' interviews before rating subjects in the study.

The four measures of substance use were:

(1) The Severity of Dependence Scale (SDS; Gossop et al. 1995), a five-item self-report questionnaire designed to measure the degree of psychological dependence experienced by users 
of different types of illicit drugs (Gossop et al. 1995). It focuses on impaired control of drug use, anxiety about use, and perceived difficulty in stopping.

(2) The Drug Abuse Screening Test (DAST; Skinner, 1982), a 20-item self-report screening instrument designed to identify individuals who have a drug use problem (excluding alcohol). Designed to assess the extent of problems related to drug misuse, the instrument relates to the individual's use of drugs; physical (withdrawal symptoms) and medical complications; and emotional and personal problems associated with drug use (Skinner, 1982; Gavin et al. 1989). A cut-off score of 6 or 7 indicates a diagnosis of drug use disorder. The screening test was used as an outcome measure by measuring drug use over the previous 3 months.

(3) Alcohol Use Disorder Identification Test (AUDIT; Dawe \& Mattick, 1997), a 10-item self-report instrument designed to screen for a range of drinking problems, in particular hazardous and harmful consumption. A score of $8-10$ is associated with harmful drinking, and a score of 13 or more is likely to indicate alcohol dependence (Dawe \& Mattick, 1997).

(4) Polydrug Use was calculated by adding the total number of substances used from the Opiate Treatment Index (Darke et al. 1992) which includes alcohol, cannabis, amphetamine, cocaine, hallucinogens, inhalants and heroin (range 1-7).

Diagnoses according to ICD-10 criteria were generated using the Operational Criteria Checklist (OPCRIT; McGuffin et al. 1991), using all available clinical information. Patient hospitalization status was recorded at intake into the treatment programme and at the 3-month follow-up period. Medication use was ascertained from the treating medical officer, checked against pharmacy charts and converted to Chlorpromazine equivalent doses, using the British National Formulary guidelines (British Medical Association, 2002). Duration of substance use and psychosis co-morbidity was measured in four increments: $1=$ less than 1 month; $2=$ between 1 month and 1 year; $3=$ between 1 and 5 years; and $4=$ more than 5 years.

\section{Study design and procedure}

Participants were recruited from May 2000 to December 2001 from the three participating sites (Fremantle Mental Health Service, Rockingham Kwinana Health Service and Peel Health Service). Participants who met selection criteria were randomized into treatment or control conditions. Randomization was performed prior to any assessments being undertaken, by allocating alternate consenting patients to either the control or intervention group.

All participants received standard community mental health services, including case management, routine outpatient services and a daytime rehabilitation programme and supported accommodation, if needed. Those randomized to the control condition received a single hourlong session of education regarding drug use, encompassing consequences of drug use for people with a psychotic illness, and harm minimization strategies; this is considered 'usual practice' in these treatment settings.

The intervention group was given a specialized dual diagnosis programme over 6 weeks that combined drug use and mental health interventions. This consisted of weekly group sessions, lasting $1 \frac{1}{2} \mathrm{~h}$, tailored specifically to the participants' stage of change according to the model of Prochasca \& DiClemente (1986), as well as their reasons for drug use according to the Substance Use Scale for Psychosis (SUSP; Spencer et al. 2002). Each group had two facilitators and no more than six participants. The intervention group was a closed cohort group where no rolling admissions were allowed into the group after treatment had commenced. Consecutive subjects after the commencement of a programme were required to wait 4-8 weeks for the next programme to begin.

The treatment manual outlined each session in detail so that core aspects of the intervention were covered with all participants. The intervention encompassed peer support, motivational enhancement strategies, harm minimization and relapse prevention paradigms. Different topics were addressed each week, in the following sequence: (1) psycho-education on drug use and mental health; (2) reasons for use; (3) reasons to change; (4) harm reduction strategies; (5) coping with high-risk situations and assertiveness training; and (6) planning for the future. The delivery of treatment was based on a treatment manual that was developed and piloted with the first group (James et al. 2002). This manual served as the guide for all future groups. 


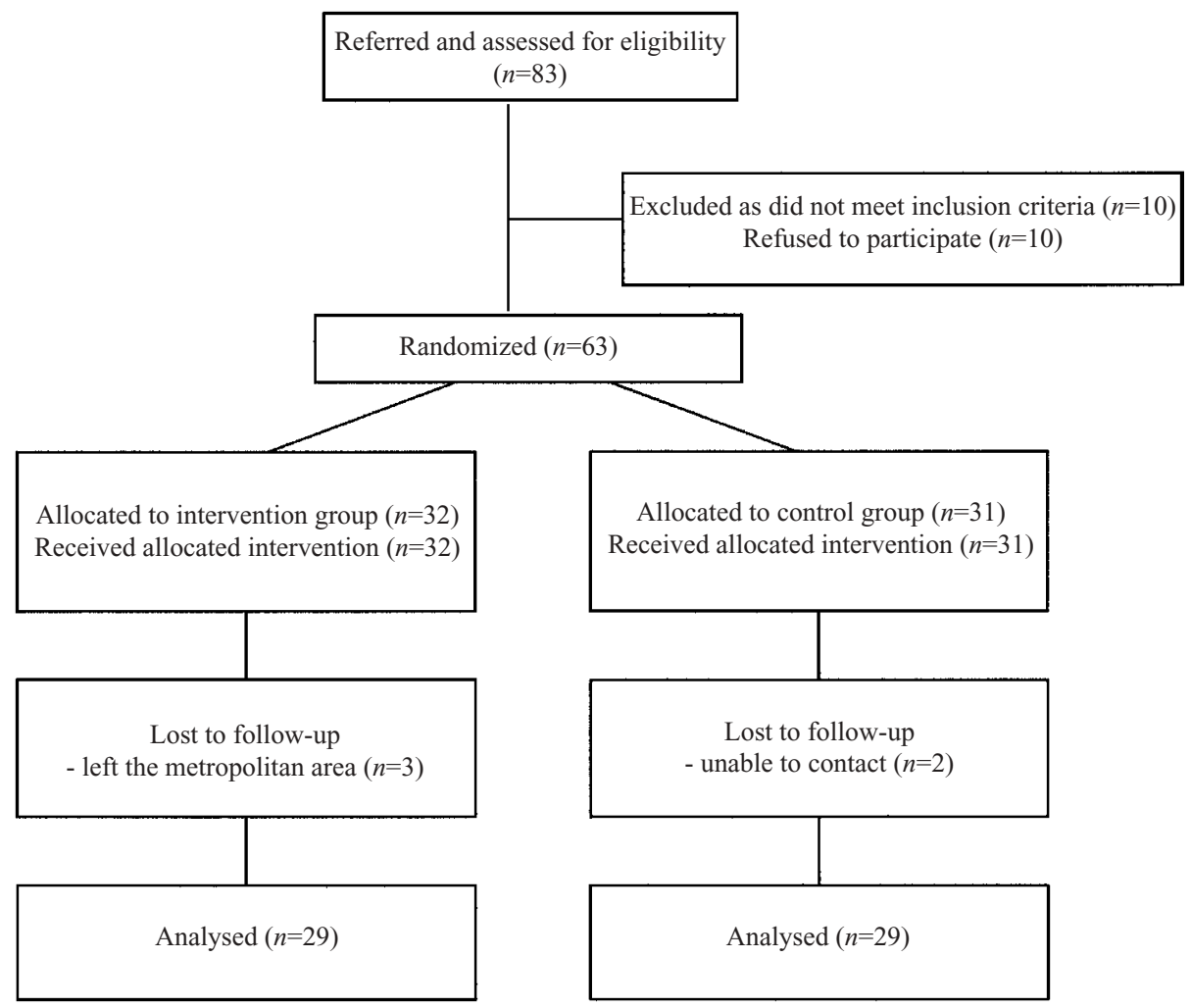

FIG. 1. Flow diagram of subjects' progress through the trial.

Treatment fidelity was ensured by strict adherence to the manual, as well as one of the originators of the manual (W.J. or G.K.) cofacilitating all subsequent groups. Before (and after) randomization, all subjects had received standard community-based treatment for their mental health problem.

Subjects were assessed at intake and 3 months after receiving their final group session. Controls were assessed in the same time frame. An independent rater (N.P.) made all follow-up assessments, 'blind' to the randomization outcome or treatment received by subjects.

\section{Analysis}

Participant demographics, length of co-morbidity, mental health service use, psychopathology, chlorpromazine equivalent dose and substance use at baseline were assessed for statistical equivalence between intervention and control subjects. A two-tailed repeated measures analysis of variance under the General Linear
Model (SPSS v. 11.00) was used for psychopathology and substance use variables from intake to 3 months. Time by group interactions were examined. Post-hoc paired $t$ tests were used to indicate which group contributed to change over the treatment period. Wilcoxon Signed Rank tests were used to examine hospitalization status of subjects in each group from intake to follow-up.

\section{Power calculations}

Because of the limited number of studies in this area, we based our initial power calculations on the basis of clinically significant $(20 \%)$ change on the main rating scales employed (see above). We were also able to perform a post-hoc power calculation based on the measures of overall functioning used by Barrowclough et al. (2001) in their randomized controlled experimental study of a one to one treatment programme for patients with dual diagnosis (published after our trial had commenced). Using COMPARE2 in 
Table 1. Tests for pre-treatment equivalence between Treatment and Control groups

\begin{tabular}{|c|c|c|c|c|}
\hline & Treatment & Controls & Test & Sig. \\
\hline \multicolumn{5}{|l|}{ Demographics } \\
\hline Age & $28 \cdot 50( \pm 7 \cdot 06) *(n=32)$ & $26 \cdot 87( \pm 8 \cdot 32)(n=31)$ & $t=0.838$ & $p=0.405$ \\
\hline Male & $23(71.9 \%)$ & $22(71 \cdot 0 \%)$ & $\chi^{2}=0.006$ & $p=0.936$ \\
\hline Female & $9(28 \cdot 1 \%)$ & $9(29 \cdot 0 \%)$ & & \\
\hline Schizophrenia & $20(62.5 \%)$ & $16(51 \cdot 6 \%)$ & $\chi^{2}=0.762$ & $p=0.383$ \\
\hline Other Psychotic Disorders & $12(37 \cdot 5 \%)$ & $15(48 \cdot 4 \%)$ & & \\
\hline $\begin{array}{l}\text { Co-morbidity } \\
\quad(\text { range } 1=<1 \text { month } ; 2=1 \text { month }-1 \text { year; } \\
\quad 3=1-5 \text { years } ; 4=5 \text { years }+ \text { ) }\end{array}$ & $3 \cdot 65( \pm 0 \cdot 60)$ & $3 \cdot 48( \pm 0 \cdot 78)$ & $t=-0 \cdot 974$ & $p=0 \cdot 334$ \\
\hline Mental Health Service use (in months) & $53 \cdot 80( \pm 55 \cdot 47)(n=31)$ & $51 \cdot 22( \pm 63 \cdot 23)(n=30)$ & $t=-0 \cdot 407$ & $p=0.685$ \\
\hline \multicolumn{5}{|l|}{ Psychopathology } \\
\hline BPRS (range 18-126) & $35 \cdot 28( \pm 10 \cdot 56)(n=32)$ & $34 \cdot 29( \pm 8 \cdot 48)(n=31)$ & $t=0 \cdot 410$ & $p=0.684$ \\
\hline GSI (range $0-4$ ) & $1 \cdot 34( \pm 0 \cdot 81)(n=32)$ & $1 \cdot 40( \pm 0 \cdot 89)(n=31)$ & $t=0 \cdot 014$ & $p=0.989$ \\
\hline $\begin{array}{l}\text { Chlorpromazine equivalent dose } \\
\text { (range } 0-1500 \mathrm{mg} \text { ) }\end{array}$ & $460 \cdot 03( \pm 329 \cdot 81)(n=30)$ & $402 \cdot 26( \pm 275 \cdot 05)(n=31)$ & $t=0 \cdot 748$ & $p=0.457$ \\
\hline \multicolumn{5}{|l|}{ Substance use } \\
\hline SDS (range 0-18) & $7 \cdot 41( \pm 3 \cdot 67)(n=31)$ & $6 \cdot 75( \pm 3 \cdot 46)(n=29)$ & $t=0.716$ & $p=0 \cdot 477$ \\
\hline DAST (range $0-20$ ) & $11 \cdot 26( \pm 5 \cdot 05)(n=30)$ & $9 \cdot 10( \pm 4 \cdot 66)(n=29)$ & $t=1 \cdot 707$ & $p=0.093$ \\
\hline AUDIT (range $0-40$ ) & $11 \cdot 68( \pm 10 \cdot 19)(n=29)$ & $12.65( \pm 8.22)(n=29)$ & $t=-0.397$ & $p=0.693$ \\
\hline POLYTOT (range 1-7) & $2 \cdot 15( \pm 0 \cdot 67)(n=32)$ & $2 \cdot 09( \pm 0 \cdot 70)(n=31)$ & $t=-0 \cdot 343$ & $p=0.733$ \\
\hline
\end{tabular}

* Standard deviations indicated in parentheses $( \pm)$; BPRS, Brief Psychiatric Rating Scale; GSI, Global Severity Index from the Brief Symptom Inventory; SDS, Severity of Dependence Scale; DAST, Drug Abuse Screening Test; AUDIT, Alcohol Use Disorder Identification Test; POLYTOT, number of different substances used. Mental Health Service use analysis performed on square root transformations, raw scores presented.

the WINPEPI program (Abramson \& Gahlinger, 2001), we estimated we would require a minimum of 29 subjects in each group to have an $80 \%$ chance of detecting a difference at the $95 \%$ significance level.

\section{RESULTS}

\section{Patient characteristics}

The overall structure of the study is depicted in Fig. 1. Eighty-three subjects were assessed for eligibility. Nine subjects were excluded as they did not have schizophrenia, schizophrenia spectrum or bipolar affective disorder, and one because of being too psychiatrically unwell. Of subjects meeting inclusion criteria $(n=73), 10$ subjects refused to participate in the study after being offered an appointment for assessment. Sixty-three subjects were therefore assessed at intake into the study, all of whom were randomized to the intervention and control groups (32 and 31 respectively). Twenty-nine subjects in each group were followed-up at 3 months $(n=58)$. This represented $79 \%$ of subjects eligible to participate in the study and $92 \%$ of those allocated to the intervention and control groups. On average, subjects in the intervention group attended $4 \cdot 7$ of the six sessions.
In the intervention group, patients with schizophrenia accounted for $63 \%$ of the sample, with severe depression with psychotic symptoms accounting for $3 \%$, mania with psychosis, $6 \%$, schizoaffective disorder, $22 \%$ and 'other nonorganic psychotic disorders', $6 \%$. The control group, the respective proportions were $52 \%$ with schizophrenia, $6 \%$ with severe depression with psychotic symptoms, $29 \%$ with schizoaffective disorder, and $13 \%$ with other nonorganic psychotic disorders. Both groups were equivalent in terms of demographics, duration of co-morbidity, mental health service use, psychopathology, chlorpromazine equivalent dose and baseline substance use, indicating pretreatment equivalence between the samples (Table 1).

\section{Treatment outcome at 3-month follow-up}

Repeated measures analysis of variance indicated significant group by time interactions for psychopathology on the BPRS, substance use on the DAST and chlorpromazine equivalent dose (Table 2).

Post-hoc paired $t$ tests indicated that the intervention group showed significantly greater improvement in psychopathology $(t=2 \cdot 04, \mathrm{df}=$ $1,28, p=0 \cdot 050)$, drug abuse $(t=6 \cdot 45, \mathrm{df}=1,28$, 
Table 2. Repeated Measures Analysis of Variance of dependent variables with Group and Time interaction

\begin{tabular}{|c|c|c|c|c|c|}
\hline \multirow[b]{3}{*}{ Dependent variables } & \multirow[b]{3}{*}{ Group } & \multicolumn{2}{|l|}{ Fixed factors } & \multirow{2}{*}{\multicolumn{2}{|c|}{ Interaction effect }} \\
\hline & & \multicolumn{2}{|c|}{ Time } & & \\
\hline & & Intake & 3 months & $F$ & Group $\times$ time \\
\hline \multicolumn{6}{|l|}{ Psychopathology } \\
\hline \multirow{2}{*}{ BPRS (range 18-126) } & Treatment $(n=29)$ & $35.41( \pm 11 \cdot 08) \dagger$ & $30 \cdot 48( \pm 10 \cdot 68)$ & $7 \cdot 364$ & $* *$ \\
\hline & Controls $(n=29)$ & $33.93( \pm 8.61)$ & $38 \cdot 10( \pm 11 \cdot 54)$ & & \\
\hline \multirow[t]{2}{*}{ GSI (range 0-4) } & Treatment $(n=31)$ & $1 \cdot 36( \pm 0 \cdot 88)$ & $0.87( \pm 0.63)$ & $0 \cdot 865$ & N.S. \\
\hline & Controls $(n=29)$ & $1 \cdot 27( \pm 0.63)$ & $1 \cdot 12( \pm 77)$ & & \\
\hline \multirow{2}{*}{ POLYTOT (range 1-7) } & Treatment $(n=31)$ & $2 \cdot 16( \pm 0 \cdot 68)$ & $1.67( \pm 0.83)$ & $3 \cdot 208$ & N.S. \\
\hline & Controls $(n=29)$ & $2 \cdot 03( \pm 0 \cdot 62)$ & $2 \cdot 00( \pm 0 \cdot 84)$ & & \\
\hline \multirow[t]{2}{*}{ DAST (range 0-20) } & Treatment $(n=29)$ & $11.58( \pm 4.82)$ & $4.96( \pm 4.49)$ & $16 \cdot 619$ & $* * *$ \\
\hline & Controls $(n=29)$ & $9 \cdot 10( \pm 4 \cdot 66)$ & $8 \cdot 24( \pm 5 \cdot 31)$ & & \\
\hline \multirow[t]{2}{*}{ CPZ (range $0-1500 \mathrm{mg}$ ) } & Treatment $(n=28)$ & $458 \cdot 73( \pm 338 \cdot 08)$ & $369 \cdot 38( \pm 357 \cdot 33)$ & $7 \cdot 221$ & ** \\
\hline & Controls $(n=27)$ & $414 \cdot 95( \pm 280 \cdot 37)$ & $514 \cdot 79( \pm 327 \cdot 40)$ & & \\
\hline \multirow{2}{*}{ AUDIT (range 0-40) } & Treatment $(n=29)$ & $11 \cdot 65( \pm 10 \cdot 19)$ & $8 \cdot 34( \pm 8 \cdot 35)$ & $1 \cdot 352$ & N.S. \\
\hline & Controls $(n=29)$ & $12 \cdot 65( \pm 8 \cdot 22)$ & $11 \cdot 65( \pm 7 \cdot 35)$ & & \\
\hline \multirow[t]{2}{*}{ SDS (range 0-18) } & Treatment $(n=28)$ & $7 \cdot 42( \pm 3.63)$ & $3.85( \pm 3.30)$ & $2 \cdot 205$ & N.S. \\
\hline & Controls $(n=29)$ & $6 \cdot 75( \pm 3.46)$ & $5 \cdot 20( \pm 3 \cdot 82)$ & & \\
\hline
\end{tabular}

$\dagger$ Standard deviations indicated in parentheses $( \pm)$, N.S., not significant, $* * p<0 \cdot 01 ; * * * p<0 \cdot 001$. BPRS, Brief Psychiatric Rating Scale; GSI, Global Severity Index from the Brief Symptom Inventory; SDS, Severity of Dependence Scale; POLYTOT, number of different substances used; DAST, Drug Abuse Screening Test; CPZ, Chlorpromazine Equivalent Dose; AUDIT, Alcohol Use Disorder Identification Test.

$p=0 \cdot 001)$, and need for medication as expressed in chlorpromazine dose equivalents $(t=2 \cdot 08$, $\mathrm{df}=1,28, p=0 \cdot 046)$. They also showed statistically greater reductions in cannabis use $(t=2 \cdot 59, \mathrm{df}=1,15, p=0 \cdot 020)$, poly substance use $(t=2 \cdot 61, \mathrm{df}=1,30, p=0 \cdot 014)$, alcohol use $(t=2.59, \mathrm{df}=1,28, p=0.015)$ and severity of dependence $(t=4 \cdot 27, \mathrm{df}=1,27, p=0 \cdot 001)$, than controls.

In comparison to the control group, patients who received the intervention had a lower rate of hospitalization at follow-up $(Z=-2 \cdot 496$, $p=0.013 ; Z=-2.837, p=0.005)$. From the intervention group, of the 11 subjects who were hospitalized at commencement of treatment (negative rank), all patients stayed out of hospital during the 3-month follow-up period. Only two subjects (positive rank) who were not hospitalized at intake required hospitalization at 3 months. From the control group, 17 subjects were hospitalized at intake and not at 3 months while six subjects who were not hospitalized at intake required hospitalization at 3 months. While more subjects were hospitalized at intake from the control group $\left(\chi^{2}=4 \cdot 73, \mathrm{df}=1, p=\right.$ 0.032 ), three times more subjects also required hospitalization at 3 months compared to those who received the group treatment.

\section{DISCUSSION}

\section{Comparison with previous work}

There remains a major deficit in terms of defining the most effective content, shape and form of interventions that will assist people with substance use co-morbidity control their substance use. Drake et al. (1998) reviewed published studies in the area and identified a number of methodological flaws, including non-randomized non-controlled experimental designs, low numbers of participants, lack of standardized assessment instruments, and complex heterogeneous interventions that were sometimes modified over the course of the trial (see James $\&$ Castle, 2003). An exception is the recent study by Barrowclough et al. (2001), which used a randomized controlled experimental design to evaluate an integrated treatment programme and resulted in a reduction in positive psychotic symptoms and an increase in the proportion of days abstinent from drugs, in a dual diagnosis group. However, the number of subjects was low (17 at end point in the treatment group and 15 in the control group), and the fact that the intervention incorporated motivational interviewing, cognitive behaviour therapy and family or caregiver intervention conducted over 
a 9-month period, raises queries about the practicability of generalizing the package for use in routine mental health service settings.

The study presented here addresses many of the shortfalls of previous studies by implementing a carefully articulated intervention, with good treatment fidelity and which is possible to implement in mental health settings without major resource implications. We used a randomized controlled trial (RCT) design and validated assessments performed 'blind' to randomization.

Our findings support the view that integrated treatment is effective for people with psychotic disorders whose illness is complicated by substance use (Drake et al. 1998; Siegfried, 1998; Drake \& Mueser, 2000; Department of Health, 2002). Integrated interventions incorporate aspects from both mental health and substance use treatments and are delivered simultaneously by the same personnel (Ley et al. 2001). We have shown that this approach can be employed in a group-based intervention within routine clinical service settings.

\section{Strengths of the study}

To our knowledge this is the first RCT of a group intervention for this population explicitly incorporating individuals' motivations for substance use (Test et al. 1989; Dixon et al. 1991; Warner et al. 1994; Baigent et al. 1995; Mueser et al. 1995; Addington \& Duchak, 1997; Fowler et al. 1998; Spencer et al. 2002). Our approach, therefore, integrated the findings of the existing literature on interventions for dual diagnosis with an intervention that targets people's reasons for use. These include using drugs and alcohol to cope with negative affect, for social affiliation and for enhancement purposes (Test et al. 1989; Dixon et al. 1991; Warner et al. 1994; Baigent et al. 1995; Mueser et al. 1995; Addington \& Duchak, 1997; Fowler et al. 1998; Spencer et al. 2002). We were also able to demonstrate that pre-treatment conditions on patient characteristics, chlorpromazine equivalent dose and psychopathology were the same between the two groups indicating an effective randomization process prior to treatment.

Our retention rates are higher than in most other studies in the field (e.g. Barrowclough et al. 2001). We believe this was due to a number of factors, including the fact that subjects gave informed consent to participate in a group-based intervention, and were thus clearly motivated to address their substance use. Also, we followed up participants between groups with reminder phone calls, and worked closely with individual case managers such that they encouraged attendance at the groups and followup assessments. Finally, all subjects were paid to attend the follow-up assessments.

\section{Limitations of the study}

Limitations of the study include small numbers and the impossibility of blinding participants and group facilitators to intervention status. We could also not control for time spent in the group setting, overall therapist contact time, nor did we conduct an independent evaluation of treatment fidelity. Furthermore, we assessed only relatively short-term outcomes. The selection process would not have introduced systematic bias, but does raise questions about generalizabity, in that we were clearly targeting a motivated group of patients. The randomization procedure was on the basis of alternative allocation of referred subjects to each arm of the study; this is perhaps not strictly 'random' allocation, but the equivalence of the groups in terms of baseline characteristics suggests it was adequate. We were reliant on self-report measures, given the relative paucity of published research on the psychometrics of substance use measures amongst patients with psychotic disorders. In addition, we were unable to confirm levels of drug or alcohol use using blood or urine samples; however, we do not suspect any systematic bias in this regard, and the fact that (objective) psychopathology ratings improved with reduced reported substance use lends support to the veracity of the findings.

Clearly this work requires replication in different and larger samples of patients from different settings, and by researchers who were not originators of the treatment package.

\section{ACKNOWLEDGEMENTS}

This project was funded by the Health Department of Western Australia. S.R.K. is currently at the Department of Psychiatry, Dalhousie University, Queen Elizabeth II Centre, Halifax, Canada. 


\section{REFERENCES}

Abramson, J. H. \& Gahlinger, P. M. (2001). Computer Programs for Epidemiologists: PEPI version 4. Sagebrush Press: Salt Lake City, Utah, USA [www.sagebrushpress.com/pepibook.html].

Addington, J. \& Duchak, V. (1997). Reasons for substance use in schizophrenia. Acta Psychiatrica Scandinavica 96, 329-333.

Baigent, M., Holme, G. \& Hafner, R. J. (1995). Self-reports of the interaction between substance abuse and schizophrenia. Australian and New Zealand Journal of Psychiatry 29, 69-74.

Barrowclough, C., Haddock, G., Tarrier, N., Lewis, S., Moring, J. O'Brien, R., Schofield, N. \& McGovern, J. (2001). Randomized controlled trial of motivational interviewing, cognitive behavior therapy, and family intervention for patients with co-morbid schizophrenia and substance use disorders. American Journal of Psychiatry 158, 1706-1713.

Bond, G. R., McDonel, E. C., Miller, L. D. \& Pensec, M. (1991). Assertive community treatment and reference groups: an evaluation of their effectiveness for young adults with serious mental illness and substance abuse problems. Psychosocial Rehabilitation Journal 15, 31-43.

British Medical Association (2002). British National Formulary. BMJ Books: London.

Darke, S., Hall, W., Wodak, A., Heather, N. \& Ward, J. (1992). Development and validation of a multi-dimensional instrument for assessing outcome of treatment among opiate users: the Opiate Treatment Index. British Journal of Addiction 87, 733-742.

Dawe, S., Loxton, N., Hides, L., Kavanagh, D. \& Mattick, R. P. (2002). Review of Diagnostic Screening Instruments for Alcohol and Other Drug Use and Other Psychiatric Disorders (2nd edn). Australian Government Publishing Service: Canberra [http:// www.health.gov.au/pubhlth/publicat/document/mono48.pdf].

Dawe, S. \& Mattick, R. P. (1997). Review of Diagnostic Screening Instruments for Alcohol and Other Drug Use and Other Psychiatric Disorders. Australian Government Publishing Service: Canberra.

Department of Health (2002). Mental Health Policy Implementation Guide: Dual Diagnosis Good Practice Guide. Department of Health Publications: London.

Derogatis, L. R. (1993). BSI: Brief Symptom Inventory. National Computer Systems: Minneapolis.

Dixon, L. (1999). Dual diagnosis of substance abuse in schizophrenia: prevalence and impact on outcomes. Schizophrenia Research 35, 93-100.

Dixon, L., Haas, G., Weiden, P. J., Sweeney, J. \& Frances, A. J. (1991). Drug abuse in schizophrenic patients: clinical correlates and reasons for use. American Journal of Psychiatry 148, 224.

Drake, R. E., Mercer-McFadden, C., Mueser, K. T., McHugo, G. J. \& Bond, G. R. (1998). Review of integrated mental health and substance abuse treatment for patients with dual disorders. Schizophrenia Bulletin 24, 589-608.

Drake, R. E. \& Mueser, K. T. (2000). Psychosocial approaches to dual diagnosis. Schizophrenia Bulletin 26, 105-118.

Fowler, I. L., Carr, V. J., Carter, N. T. \& Lewin, T. J. (1998). Patterns of current and lifetime substance abuse in schizophrenia. Schizophrenia Bulletin 24, 443-445.

Gavin, D. R., Ross, H. E. \& Skinner, H. A. (1989). Diagnostic validity of the Drug Abuse Screening Test in the assessment of DSM-III drug disorders. British Journal of Addiction 84, 301-307.

Gossop, M., Darke, S., Griffiths, P., Hando, J., Powis, B., Hall, W. \& Strang, J. (1995). The Severity of Dependence Scale
(SDS): psychometric properties of the SDS in English and Australian samples of heroin, cocaine and amphetamine users. Addiction 90, 607-614.

James, W. \& Castle, D. (2003). Substance abuse co-morbidity in schizophrenia. In Pharmacological and Psychosocial Treatments for Schizophrenia (ed. D. J. Castle, D. Copolov and T. Wykes), pp. 75-87. Martin Dunitz: London.

James, W., Koh, G., Spencer, C., Preston, N., Kisely, S. \& Castle, D. (2002). Managing Mental Health \& Drug Use. Uniprint: Perth, Western Australia.

Krausz, M., Haasen, C., Mass, R., Wagner, H. B., Peter, H. \& Freyberger, H. S. (1996). Harmful use of psychotropic substances by schizophrenics: coincidence, patterns of use and motivation. European Addiction Research 2, 11-16.

Ley, A., McLaren, S. \& Siegfried, N. (2001). Treatment programmes for people with both severe mental illness and substance misuse (Cochrane Review). Cochrane Library, Issue 1.

Martinez-Aravelo, M. J., Calcedo-Ordonez, A. \& Varo-Prieto, J. R. (1994). Cannabis consumption as a prognostic factor in schizophrenia. British Journal of Psychiatry 164, 679-681.

McGuffin, P., Farmer, A. \& Harvey, I. (1991). A polydiagnostic application of operational criteria in studies of psychotic illness. Development and reliability of the OPCRIT system. Archives of General Psychiatry 48, 764-770.

Mueser, K. T., Nishith, P., Tracy, J. L., DeGirolamo, L. \& Molinaro, M. (1995). Expectations and motives for substance use in schizophrenia. Schizophrenia Bulletin 21, 367-378.

Overall, J. E. \& Gorham, D. R. (1962). The brief psychiatric rating scale. Psychological Reports 10, 799-812.

Prochasca, J. \& DiClemente, C. (1986). Toward a comprehensive model of change. In Treating Addictive Behaviours: Process of Change (ed. L. D. Miller \& N. Heather), pp. 3-27. Plenum: New York.

Regier, D. A., Farmer, M. E., Rae, D. S., Locke, B. Z., Keith, S. J., Judd, L. L. \& Goodwin, F. K. (1990). Comorbidity of mental disorders with alcohol and other drug abuse. Journal of the American Medical Association 264, 2511-2518.

Schneier, F. R. \& Siris, S. G. (1987). A review of psychoactive substance abuse and schizophrenia: patterns of drug choice. Journal of Nervous and Mental Disease 175, 641-652.

Siegfried, N. (1998). A review of comorbidity: major mental illness and problematic substance use. Australian and New Zealand Journal of Psychiatry 32, 707-717.

Spencer, C., Castle, D. \& Michie, P. (2002). An examination of the validity of a motivational model for understanding substance use among individuals with psychotic disorders. Schizophrenia Research 28, 233-247.

Strakowski, S. M., McElroy, S. L., Keck, P. W. \& Wesy, S. A. (1994). The co-occurrence of mania with medical and other psychiatric disorders. International Journal of Psychiatry Medicine 22, 305-328.

Skinner, H. A. (1982). The drug abuse screening test. Addictive Behaviours 7, 363-371.

Test, M. A., Wallisch, L. S., Allness, D. J. \& Ripp, K. (1989) Substance abuse in young adults with schizophrenic disorders. Schizophrenia Bulletin 15, 465-476.

Warner, M. B., Taylor, D., Wright, J., Sloat, A., Springet, G., Arnold, S. \& Weinberg, H. (1994). Substance use among the mentally ill: prevalence, reasons for use and effects on illness. American Journal of Orthopsychiatry 64, 30-39.

WHO (1994). The ICD-10 Classification of Mental and Behavioural Disorders. World Health Organisation: Geneva. 


\section{University Library}

\section{- M M N E R VA A gateway to Melbourne's research publications}

Minerva Access is the Institutional Repository of The University of Melbourne

Author/s:

James, W;Preston, NJ;Koh, G;Spencer, C;Kisely, SR;Castle, DJ

Title:

A group intervention which assists patients with dual diagnosis reduce their drug use: a randomized controlled trial

Date:

2004-08-01

Citation:

James, W., Preston, N. J., Koh, G., Spencer, C., Kisely, S. R. \& Castle, D. J. (2004). A group intervention which assists patients with dual diagnosis reduce their drug use: a randomized controlled trial. PSYCHOLOGICAL MEDICINE, 34 (6), pp.983-990. https://doi.org/10.1017/ S0033291703001648.

Publication Status:

Published

Persistent Link:

http://hdl.handle.net/11343/33029 